\title{
ARTICLE
}

Acute myeloid leukemia

\section{The clinical mutatome of core binding factor leukemia}

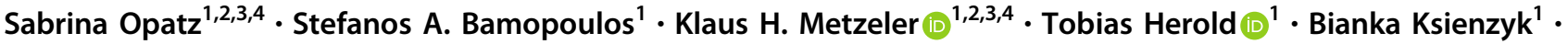 \\ Kathrin Bräundl ${ }^{1,2,3,4}$. Sebastian Tschuri ${ }^{1,3,4} \cdot$ Sebastian Vosberg ${ }^{2} \cdot$ Nikola P. Konstandin $^{1} \cdot$ Christine Wang $^{1}$. \\ Luise Hartmann ${ }^{1,2,3,4}$. Alexander Graf ${ }^{5}$ - Stefan Krebs ${ }^{5}$ - Helmut Blum ${ }^{5}$ - Stephanie Schneider ${ }^{1,6}$. \\ Christian Thiede ${ }^{3,4,7} \cdot$ Jan Moritz Middeke ${ }^{3,4,7}$. Friedrich Stölzel ${ }^{3,4,7}$. Christoph Röllig ${ }^{3,4,7} \cdot$ Johannes Schetelig ${ }^{3,4,7}$. \\ Gerhard Ehninger, ${ }^{3,4}$, Alwin Krämer ${ }^{3,4} \cdot$ Jan Braess $^{8}$ - Dennis Görlich ${ }^{9} \cdot$ Maria Cristina Sauerland ${ }^{9}$. \\ Wolfgang E. Berdel ${ }^{10} \cdot$ Bernhard J. Wörmann $^{11}$ - Wolfgang Hiddemann ${ }^{1,2,3,4} \cdot$ Karsten Spiekermann $^{1,2,3,4}$. \\ Stefan K. Bohlander (iD) ${ }^{12}$ Philipp A. Greif ${ }^{1,2,3,4}$
}

Received: 23 July 2019 / Revised: 28 November 2019 / Accepted: 12 December 2019 / Published online: 2 January 2020 (c) The Author(s) 2020. This article is published with open access

\begin{abstract}
The fusion genes CBFB/MYHI1 and RUNX1/RUNXIT1 block differentiation through disruption of the core binding factor (CBF) complex and are found in 10-15\% of adult de novo acute myeloid leukemia (AML) cases. This AML subtype is associated with a favorable prognosis; however, nearly half of CBF-rearranged patients cannot be cured with chemotherapy. This divergent outcome might be due to additional mutations, whose spectrum and prognostic relevance remains hardly defined. Here, we identify nonsilent mutations, which may collaborate with CBF-rearrangements during leukemogenesis by targeted sequencing of 129 genes in 292 adult CBF leukemia patients, and thus provide a comprehensive overview of the mutational spectrum ('mutatome') in CBF leukemia. Thereby, we detected fundamental differences between CBFB/MYH11and RUNX1/RUNXIT1-rearranged patients with ASXL2, JAK2, JAK3, RAD21, TET2, and ZBTB7A being strongly correlated with the latter subgroup. We found prognostic relevance of mutations in genes previously known to be AML-associated such as KIT, SMC1A, and DHX15 and identified novel, recurrent mutations in NFE2 (3\%), MN1 (4\%), HERC1 (3\%), and ZFHX4 $(5 \%)$. Furthermore, age $>60$ years, nonprimary AML and loss of the Y-chromosomes are important predictors of survival. These findings are important for refinement of treatment stratification and development of targeted therapy approaches in CBF leukemia.
\end{abstract}

Supplementary information The online version of this article (https:// doi.org/10.1038/s41375-019-0697-0) contains supplementary material, which is available to authorized users.

Philipp A. Greif

pgreif@med.uni-muenchen.de

1 Laboratory for Leukemia Diagnostics, Department of Medicine III, University Hospital, LMU Munich, Munich, Germany

2 Experimental Leukemia \& Lymphoma Research, Department of Medicine III, University Hospital, LMU Munich,

Munich, Germany

3 German Cancer Consortium (DKTK), Heidelberg, Germany

4 German Cancer Research Center (DKFZ), Heidelberg, Germany

5 Laboratory for Functional Genome Analysis at the Gene Center, LMU Munich, Munich, Germany

6 Institute of Human Genetics, University Hospital, LMU Munich, Munich, Germany

\section{Introduction}

The core binding factor $(\mathrm{CBF})$ protein complex, composed of RUNX1 and CBFB, is important for myeloid differentiation and recurrently altered in acute myeloid leukemia

7 Department of Internal Medicine 1, University Hospital Carl Gustav Carus, Dresden, Germany

8 Oncology and Hematology, St. John of God Hospital, Regensburg, Germany

9 Institute of Biostatistics and Clinical Research, University of Münster, Münster, Germany

10 Department of Medicine A, Hematology, Oncology and Pneumology, University of Münster, Münster, Germany

11 Department of Hematology, Oncology and Tumor Immunology, Charité University Medicine, Campus Virchow, Berlin, Germany

12 Department of Molecular Medicine and Pathology, Faculty of Medical and Health Sciences, University of Auckland, Auckland, New Zealand 
(AML) through genomic rearrangements. The fusion gene CBFB/MYHII results from a pericentric inversion of chromosome 16, inv(16)(p13.1q22), or less commonly from a $\mathrm{t}(16 ; 16)(\mathrm{p} 13.1 ; \mathrm{q} 22)$. A translocation between chromosomes 8 and 21 [t $(8 ; 21)(\mathrm{q} 22 ; \mathrm{q} 22)]$ underlies the RUNX1/ RUNX1T1 fusion. Both rearrangements disrupt CBF function, leading to a block of myeloid differentiation and ultimately to leukemia. The resulting so-called CBF leukemias constitute $10-15 \%$ of adult de novo AML cases and are associated with favorable prognosis $[1,2]$.

Mutations in the receptor tyrosine kinase genes KIT and FLT3 and the proto-oncogenes NRAS and KRAS have been observed in up to $80 \%$ of CBF leukemia patients and probably serve as cooperating factors during leukemogenesis promoting proliferation [3-6]. Identification of these additional genetic abnormalities could be helpful in predicting treatment outcome $[5,7,8]$, since $45 \%$ of CBF leukemia patients relapse after standard chemotherapy $[9,10]$.

To systematically identify those cooperating mutations, we performed whole-exome sequencing (WES) of 12 adult AML patients with CBF rearrangement.

Even though both fusion products are sharing a similar pathogenic mechanism, there is a considerable clinical, cytomorphological and molecular variability within $\mathrm{CBF}$ leukemia [8-11]. Hence, the question arises of whether patients with $\mathrm{t}(8 ; 21)$ and inv(16) leukemia should be considered distinct entities. To answer this question on the basis of the underlying spectrum of molecular differences, we compared the mutation profile (i.e. 'mutatome') of 162 patients with inv(16) leukemia to 130 patients with $t(8 ; 21)$ leukemia by targeted sequencing of 129 genes. Furthermore, we correlated distinct molecular and chromosomal aberrations with clinical outcome.

\section{Materials and methods}

\section{Patient samples}

For exome sequencing, diagnostic bone marrow (BM) samples were collected from 12 patients diagnosed with $\mathrm{CBF}$ Leukemia according to standard French-American-British and World Health Organization criteria between 2003 and 2011. The patients were selected based on availability of suitable samples. The $\operatorname{inv}(16)(\mathrm{p} 13.1 \mathrm{q} 22) \quad[n=8]$ or $\mathrm{t}(16 ; 16)(\mathrm{p} 13.1 ; \mathrm{q} 22) \quad[n=2]$ was detected by standard chromosome banding analysis and fluorescence in situ hybridization. The fusion transcripts $C B F B / M Y H 11$ type A $(n=8)$ and type $\mathrm{D}(n=2)$ were confirmed by reversetranscriptase polymerase chain reaction (RT-PCR). After intensive induction therapy, based on cytarabine and anthracyclines, complete remission (CR) was achieved in all patients ( $<5 \%$ BM blasts; $C B F B / M Y H 11$ transcripts no longer
Table 1 Patient characteristics

\begin{tabular}{|c|c|}
\hline CBF leukemia & $n=292$ \\
\hline \multicolumn{2}{|l|}{ Outcome } \\
\hline $\mathrm{CR}$ & $96 \%$ \\
\hline Relapse & $45 \%$ \\
\hline Death & $33 \%$ \\
\hline CBFB/MYH11 & $n=162$ \\
\hline $\operatorname{inv}(16)$ & $131(81 \%)$ \\
\hline $\mathrm{t}(16 ; 16)$ & $16(10 \%)$ \\
\hline$C B F B / M Y H 11$, not specified & $15(9 \%)$ \\
\hline Type A fusion & $90(83 \%)$ \\
\hline Nontype A fusion & $13(12 \%)$ \\
\hline Type D & $7(6 \%)$ \\
\hline Type E & $4(4 \%)$ \\
\hline Type S & $2(2 \%)$ \\
\hline Fusion not specified & $53(33 \%)$ \\
\hline de novo AML & $147(91 \%)$ \\
\hline t-AML & $13(8 \%)$ \\
\hline s-AML & $2(1 \%)$ \\
\hline Blasts in BM, median (range) & $62 \%(10-95 \%)$ \\
\hline Blasts in PB, median (range) & $47 \%(6-93 \%)$ \\
\hline Age in years, median (range) & $44(17-83)$ \\
\hline Male sex & $54 \%(88 / 162)$ \\
\hline Allogeneic HCT in 1 st CR & $12(7 \%)$ \\
\hline RUNX1/RUNX1T1 & $n=130$ \\
\hline de novo AML & $114(88 \%)$ \\
\hline $\mathrm{t}-\mathrm{AML}$ & $12(9 \%)$ \\
\hline s-AML & $4(3 \%)$ \\
\hline Blasts in BM, median (range) & $55 \%(10-90 \%)$ \\
\hline Blasts in PB, median (range) & $44 \%(6-78 \%)$ \\
\hline Age in years, median (range) & $54(16-79)$ \\
\hline Male sex & $60 \%(78 / 130)$ \\
\hline Allogeneic HCT in 1st CR & $12(9 \%)$ \\
\hline
\end{tabular}

detectable by RT-PCR). A matched BM sample at CR was used as normal control for exome sequencing. Exome sequencing of two patients with AML $t(8 ; 21)$ was performed as described in a previous study conducted in our laboratory [12].

For targeted gene sequencing, we used BM $(n=259)$ or peripheral blood ( $\mathrm{PB} ; n=33$ ) samples from 162 adult patients with newly diagnosed and untreated AML with $C B F B / M Y H 11$-rearrangement and from 130 patients with RUNX1/RUNX1T1-rearrangement. These 292 patients included the 12 cases initially analyzed by exome sequencing. The patient characteristics are provided in Table 1. If available, matched BM aspirates obtained during $\mathrm{CR}$ were analyzed to evaluate the somatic status of detected variants $(n=110$, $38 \%$ ). Patients were treated according to protocols of the AML Cooperative Group or Study Alliance Leukemia. Study 
protocols were approved by the ethics committees of the participating centers. Written informed consent for scientific use of surplus samples was obtained in accordance with the Declaration of Helsinki.

\section{Whole-exome sequencing}

Genomic DNA was extracted from BM or PB samples, after enrichment of mononuclear cells (Ficoll), using QIAcube technology (Qiagen, Hilden, Germany). Three micrograms of genomic DNA from diagnostic as well as matched remission BM specimen was used for preparation of sequencing libraries as described previously [3]. Protein-coding sequences were captured using SureSelect human all exon $50 \mathrm{Mb}$ kit version 3 (Agilent, Santa Clara, CA) according to the manufacturer's instructions. Exome libraries were sequenced with 76-bp paired end reads on a Genome Analyzer IIx or HiSeq platform (Illumina, San Diego, CA). Sequence alignment and variant detection was performed as described previously [13].

\section{Custom-targeted sequencing}

Candidate genes identified by exome sequencing $(n=47)$ and genes known to be recurrently mutated in AML $(n=82)$ were studied by targeted amplicon sequencing $\left(\right.$ HaloPlex $^{\circledR}$; Agilent Technologies, Santa Clara, CA) in 292 CBF leukemia patients in order to identify genes with recurring mutations. A custom gene-panel was designed using an online software (Sure Design, Agilent Technologies, Santa Clara, CA). Our panel comprises 129 genes and a total target sequence of $396.45 \mathrm{kbp}$ (Supplementary Table S1). From 110 patients-matched $\mathrm{CR}$ samples were available and sequenced to exclude germline variants. Library preparation was performed according to the manufacturer's protocol (HaloPlex ${ }^{\circledR}$ target enrichment system for Illumina sequencing, Version D.4, March 2013) using 225 ng genomic DNA per patient sample. The resulting libraries were sequenced by performing 250-bp paired end reads on a MiSeq and HiSeq instrument (Illumina, San Diego, CA) to an average target coverage of 500x. Software and detailed parameters used for variant calling were previously described [14].

\section{Clinical outcome analysis}

Statistical analyses were performed using $\mathrm{R}$ version 3.4.1 (R Foundation for Statistical Computing, Vienna, Austria). Only patients who received intensive treatment $(n=157$ [CBFB/MYH11] and $n=127$ [RUNXI/RUNXIT1]) were included in the clinical outcome analyses (median followup: 3.6 years). Twenty-four patients received an allogeneic stem cell transplantation (HSCT) in first CR. Since indications for HSCT in first CR were not uniform and HSCT significantly prolongs relapse-free survival (RFS) in our
CBF cohort (Supplementary Fig. S1), survival analyses were censored at the timepoint of transplantation in first $\mathrm{CR}$ but not later during the course of the disease. Mutations with frequencies less than $5 \%$ in the examined groups and variables with missing values in more than $15 \%$ of the patients were excluded from the analysis.

Clinical outcome was visualized using the Kaplan-Meier method and log-rank tests were calculated without adjustment of $p$ values. Correlations of inv(16) and $\mathrm{t}(8 ; 21)$ mutations were assessed using the Mann-Whitney $U$ test for measurement variables, the Pearson's chi-squared test for categorical variables and the Fisher's exact test for binary variables. All tests were two tailed. In case of multiple testing, $p$ values were adjusted using the Benjamini-Hochberg procedure. The false discovery rate cutoff was set at $<0.05$. Survival analysis was performed using Cox regression models. A multivariate Cox regression model was built using all variables with a $p$ value $<$ 0.1 in the univariate regression models. Hazard ratios (HR) with corresponding Wald-test $p$ values and 95\% confidence intervals (CI) are reported. Outcome parameters were defined according to recent European Leukemia Net recommendations [2]. All statistical tests were considered significant at $p \leq 0.05$.

\section{Results}

\section{Somatic mutation profile of $12 \mathrm{CBF}$ leukemia patients}

To systematically detect somatic mutations, we sequenced the protein-coding regions of 12 matched diagnostic and remission samples, generating at least $2.9 \mathrm{Gbp}$ of raw sequence data from each exome (mean $5.1 \mathrm{Gbp}$ ). Thereby we covered more than $80 \%$ of RefSeq coding exon positions with a minimum read depth of 10 (Supplementary Table S2). The study design is outlined in Fig. 1. We identified 1-13 somatically acquired, leukemia-specific sequence variants per patient (median $=6$ ). These include mutations in genes known to cooperate with CBF-rearrangements [e.g. NRAS $(n=6), \operatorname{KRAS}(n=4)$, FLT3 $(n=2), \operatorname{KIT}(n=3)$, ZBTB7A $(n=2)]$ as well as in genes, which have not been described to be mutated in CBF leukemia so far (e.g. ZFHX4, NFE2, HERC1). Of note, targeted amplicon sequencing with higher coverage revealed several subclonal mutations of known AML driver genes (FLT3, NRAS, and KRAS), which were missed by exome sequencing. Results are summarized in Supplementary Fig. S2 and Table S3.

\section{Additional mutations in 162 CBFB/MYH11- rearranged $A M L$ samples}

By targeted sequencing, additional molecular mutations were detected in 156/162 patients (96\%). Fifteen genes 
Fig. 1 Study design. Exome sequencing of 12 adult patients with CBF leukemia was followed by custom-targeted sequencing of candidate genes from exome sequencing and known mutational targets in diagnostic samples of 162 patients with inv(16) and 130 patients with $t(8 ; 21)$. Ultimately, the mutation profiles were compared between the two subgroups.

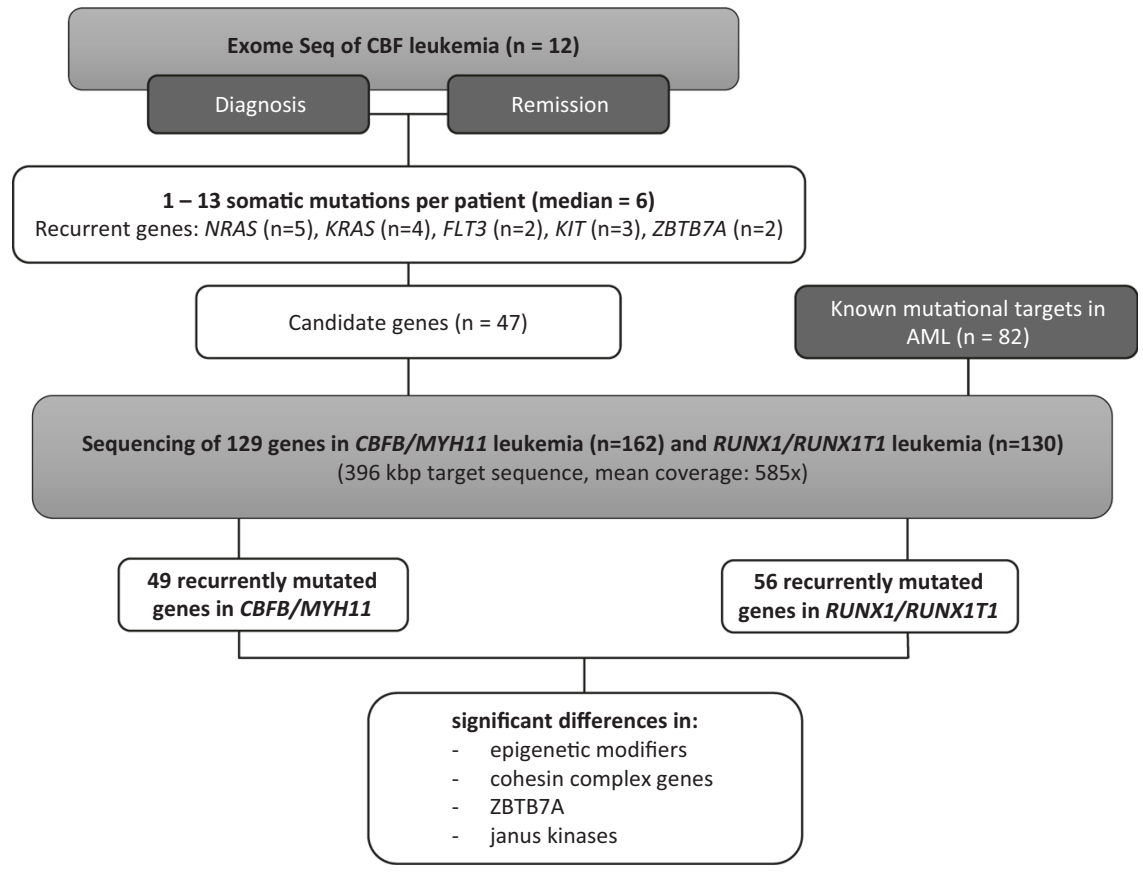

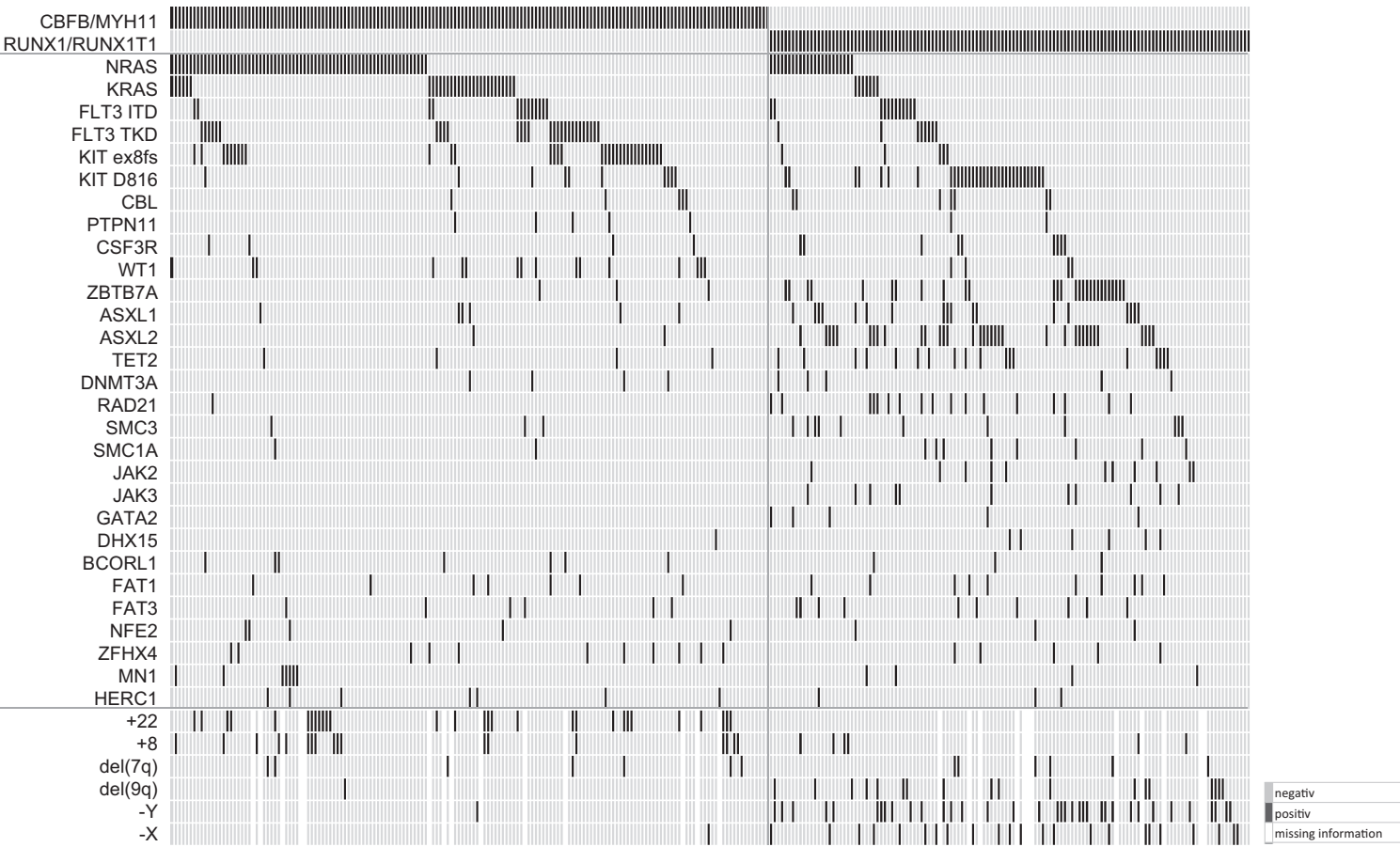

Fig. 2 Results from targeted amplicon sequencing in 162 patients with $C B F B / M Y H 11$-rearrangement (left), and 130 patients with RUNX1/RUNX1T1-rearrangement (right). Each column represents

were found mutated in at least five patients (frequency $\geq$ $3,1 \%$ ). Results are summarized in Fig. 2.

The mutation frequency of known CBF leukemia associated genes was similar to previous reports [3-6, 8]; Most one patient, each line shows the status of the indicated genetic aberrations.

common were missense mutations of $R A S$-genes, present in $57 \%$ of the patients ( $43 \% N R A S, 19 \%$ KRAS), affecting the known hotspots (G12, G13, Q61). NRAS mutations were highly associated with inv(16)-leukemia and trisomy of 
Fig. 3 Correlation of inv(16) and $t(8 ; 21)$ leukemia with alterations common in $\mathrm{CBF}$ AML. Significance levels as followed: $*=<0.05$, $* *=<0.01, * * *=<0.001$.

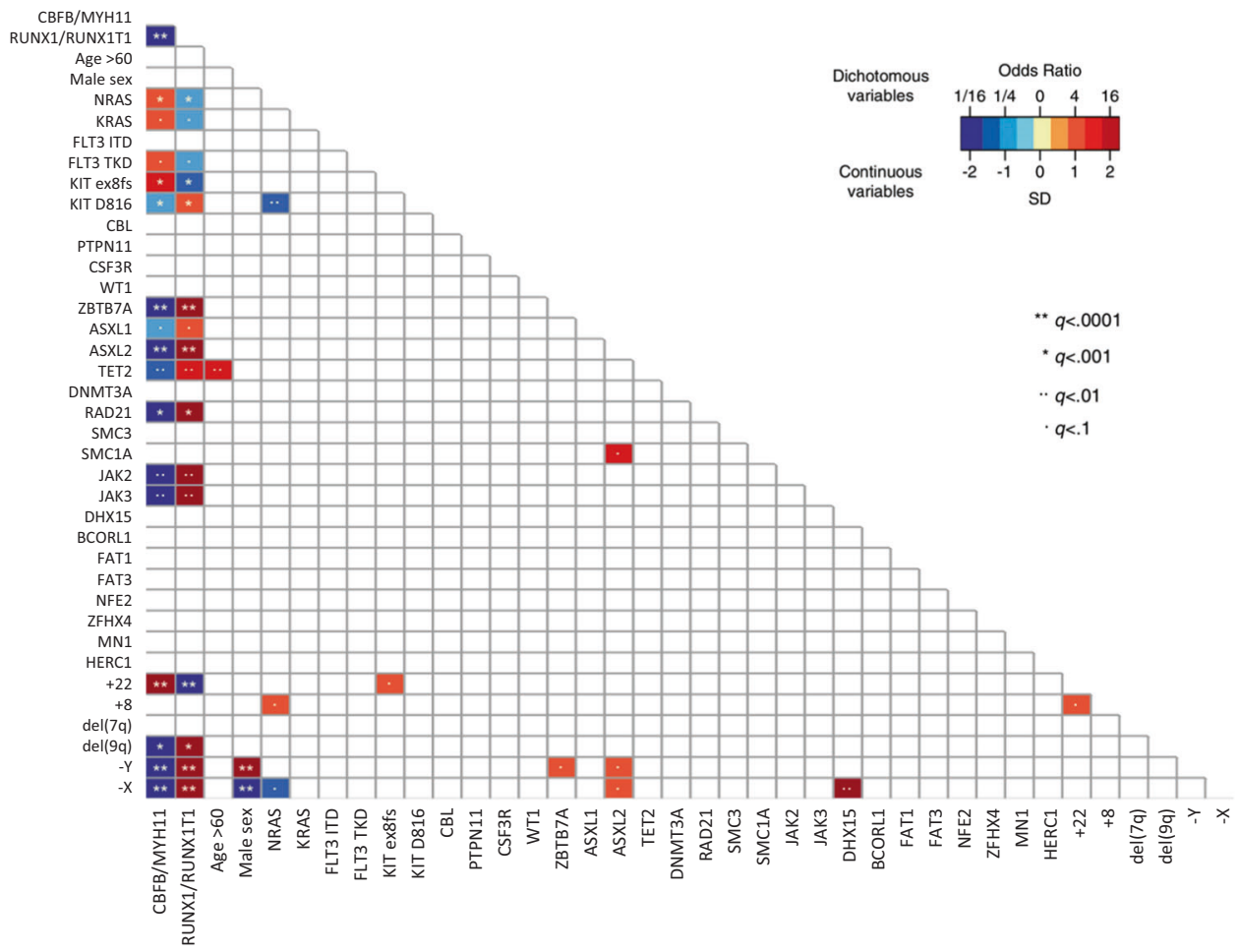

chromosome 8 but rarely found in patients with point mutations in KIT D816 (Fig. 3). Next in frequency were FLT3 mutations in $27 \%$ of the cases. FLT3-ITD mutations were less common in our cohort than in cytogenetic normal AML $(13 / 162 ; 8 \%)$, whereas point mutations or short deletions in FLT3 (e.g. D835mut $n=18$, N676K $n=8$ ) had a frequency of $18 \%$ and were associated with the inv(16) subtype. A total of $26 \%$ of the CBFB/MYH11-positive patients had a KIT mutation, with $6 \%$ D816 point mutations (10/162) and $19 \%$ exon 8 frameshift mutations (31/162). Exon 8 frameshift mutations were highly associated with CBFB/MYH11-rearranged leukemia, while point mutations affecting position D816 were correlated with RUNX1/ RUNX1T1 leukemia (Fig. 3).

\section{Additional mutations in 130 RUNX1/RUNX1T1- rearranged AML samples}

Signaling pathway alterations affecting $R A S$-genes and FLT3 are less frequent in patients with $R U N X 1 / R U N X 1 T 1$ compared with $C B F B / M Y H 11$-rearranged AML. There was no significant difference in the KIT mutation frequency, but while $K I T$ exon 8 frameshift mutations are associated with $C B F B / M Y H 11$-rearranged leukemia $(n=31)$, they are underrepresented in $R U N X 1 / R U N X 1 T 1$ leukemia $(n=5)$ and here outnumbered by KIT D816 point mutations $(n=$ 33 vs.10, 25\%; Fig. 3). Mutations in janus kinases (JAK) were identified exclusively in patients with RUNX1/ RUNX1T1-rearranged AML (16\%). Consistent with recent reports [8, 12, 15-19], we found recurrent mutations in transcription factors [ZBTB7A $(22 \%)$ and GATA2 (4\%)], chromatin modifiers [ASXL1 (14\%), ASXL2 (29\%), EZH2 (2\%) and KDM6A (2\%)], genes involved in DNA methylation [TET2 (14\%), DNMT3A (4\%), IDH1 (2\%) and IDH2 $(3 \%)]$, as well as mutations in genes that encode components of the cohesin complex [RAD21 (14\%), SMC3 (8\%), SMC1A (6\%) and STAG2 (2\%)]. These common genetic changes are rare in inv(16) cases.

In addition, we found recurrent mutations in the RNA helicase $D H X 15$ in $5 \%$ of patients $(6 / 130)$ with a mutational hotspot in the ATP-binding domain (R222; Fig. 4). Sequencing results are shown in Fig. 2.

\section{Additional mutations shared in both CBF leukemia subtypes}

Alterations of $C B L$ were found in a total of 12 patients $(4 \%)$ and PTPN11 was found mutated in 7 patients (2\%). In addition, we found recurrent $C S F 3 R$ mutations in 13 patients (5\%), with a hotspot in the extracellular fibronectin-like type VI domain (e.g. T615A and T618I) and mutations that truncate the cytoplasmic domain (e.g. S810fs). In addition to common signaling pathway mutations, we found 20 patients with WT1 mutations causing a frameshift in exon $6(7 \%)$ and 10 patients with alterations in BCORL1 (3\%).

Mutations in the FAT protocadherin family members, FAT1 and FAT3 were found in 17 and 16 patients, respectively $(6 \%)$. In addition, we identified 16 patients 

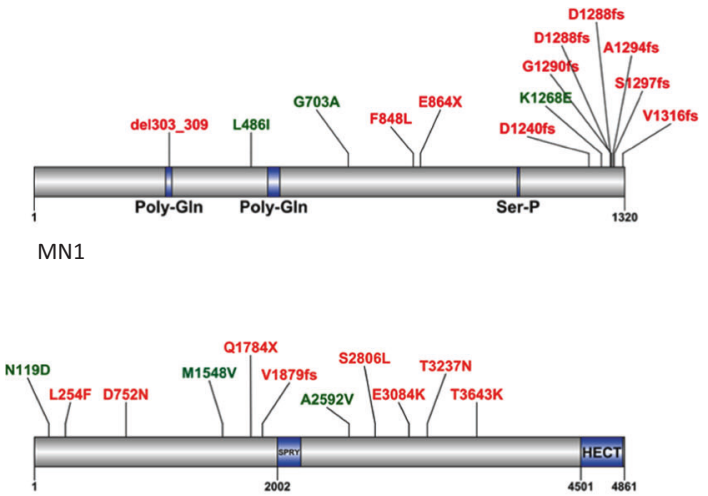

HERC1

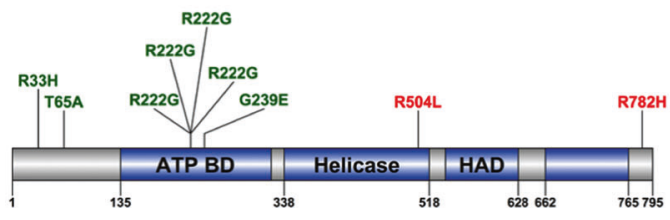

DHX15

Fig. 4 The location of mutations in the novel recurrently mutated genes MN1, NFE2, HERC1, ZFHX4 and mutational hotspots in CSF3R and DHX15. Known protein domains are shown. Mutations

with mutations in ZFHX4 (6\%), 11 patients with alterations in $M N 1$ (4\%), 8 patients with NFE2 (3\%), and 10 patients with HERC1 (3\%) mutations. Of note, 3 out of 8 NFE2 mutations are affecting position R273 in the DNA-binding domain (DBD), suggesting a mutational hotspot. Further, we identified hotspot mutations in MN1 resulting in loss of the C-terminal part (Fig. 4).

Regarding the $33 \mathrm{~PB}$ samples, the mutational pattern is similar to the mutation profile of the BM samples (Supplementary Fig. S3). Range of the PB blast count was 6-93\% (Median: 40\%, Mean: 45.6\%) and even in samples with low blast count, common mutations could be identified. This is likely due to the enrichment of mononuclear cells (including blasts) by Ficoll gradient during the sample preparation. Ultimately, the exact content of blasts in the samples analyzed by sequencing cannot be determined but is likely higher than in the initial samples evaluated by cytomorphology.

\section{Clinical relevance of recurring mutations}

There was no significant difference in OS (overall survival) or RFS between patients with RUNX1/RUNXIT1-rearranged AML or those with $C B F B / M Y H 11$-rearranged AML (Supplementary Fig. S4). Patients with CBF AML aged above 60 years had a shorter OS than younger patients $(\mathrm{HR}=2.79$ and 3.5, respectively, $p<0.01$; Fig. 5a).

The impact of mutations, chromosomal abnormalities, and clinical variables on outcome in CBF leukemia analyzed by univariate cox regression is shown in Supplementary Figs. S5 and S6. When analyzing the prognostic relevance of distinct
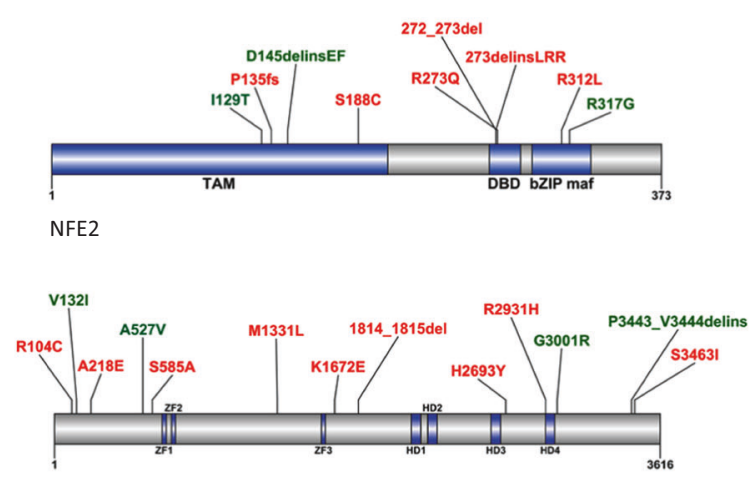

ZFHX4

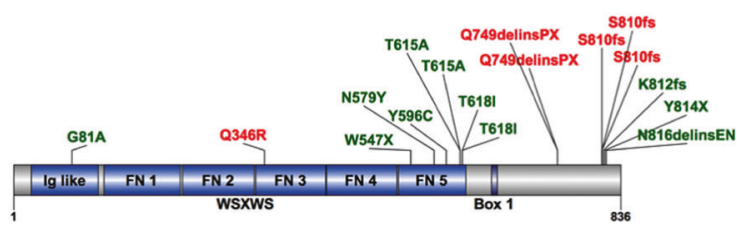

CSF3R

colored in red were found in $C B F B / M Y H 11$-rearranged samples, mutations colored in green were found in our RUNX1/RUNX1T1rearranged AML cohort.

mutations by multivariate cox regression, KIT D816, DHX15, and SMC1A mutations were independent prognostic factors (Fig. 5). Mutations in DHX15 and SMC1A correlated with shorter OS in RUNXI/RUNXIT1 leukemia (DHX15: HR = 3.57, $p=0.02$; SMC1A: HR $=6.47, p<0.001$ ), while point mutations in KIT at position D816 correlated with reduced RFS in RUNXI/RUNXIT1 leukemia ( $\mathrm{HR}=2.27, p=0.046$; Supplementary Fig. S7). In contrast to recent reports [8, 20, 21], the mutational burden of KIT D816 mutations was not relevant when assessing the prognostic impact of this mutation in our cohort (Supplementary Fig. S8). Considering the adverse prognostic relevance of SMC1A mutations, we evaluated alterations of genes encoding components of the cohesion complex together resulting in significantly shorter OS (HR $=1.93, p=0.05)$ but not RFS (HR $=0.96$, $p=0.8)$ in univariate analysis. These findings suggest that SMC1A has a unique prognostic relevance unlike the other cohesion genes.

Almost half of the CBF leukemia patients had additional chromosomal changes. Trisomy 22 was significantly associated with inv(16) leukemia, while a deletion of $9 q$ and deletion of sex chromosomes correlated with $\mathrm{t}(8 ; 21)$ leukemia. $C B F B / M Y H 11$-rearranged patients with trisomy 8 or trisomy 22 had a tendency toward longer RFS than patients without these trisomies (Fig. 5b). Loss of the Y-chromosome in male patients correlated with improved RFS in univariate analysis in RUNX1/RUNXIT1 leukemia $(\mathrm{HR}=0.39, p=0.047$; Supplementary Fig. S6). Due to the low patient number multivariate analysis could not be performed with regards to gender. 


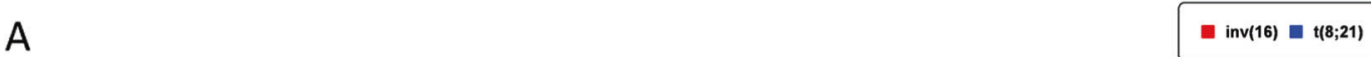

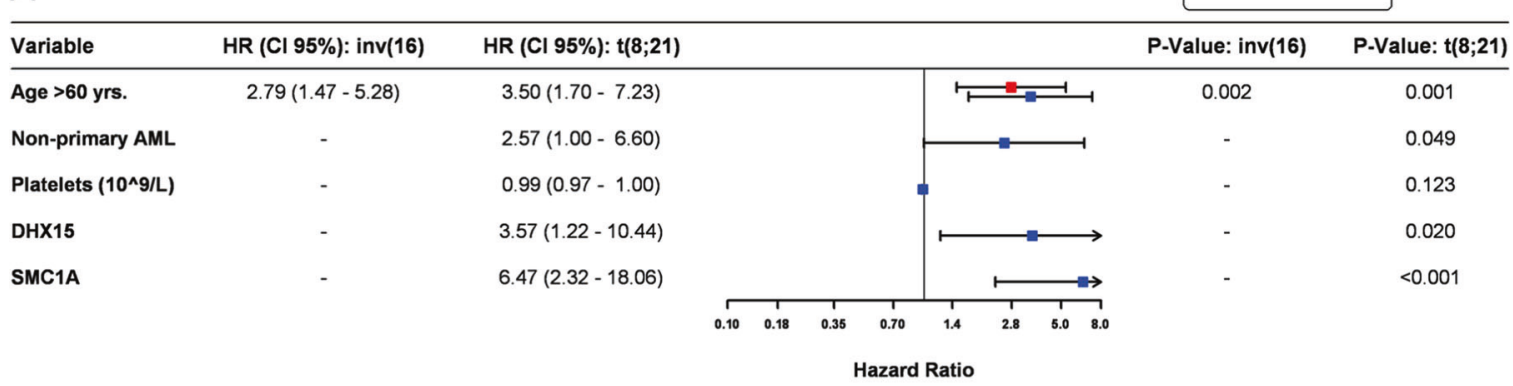

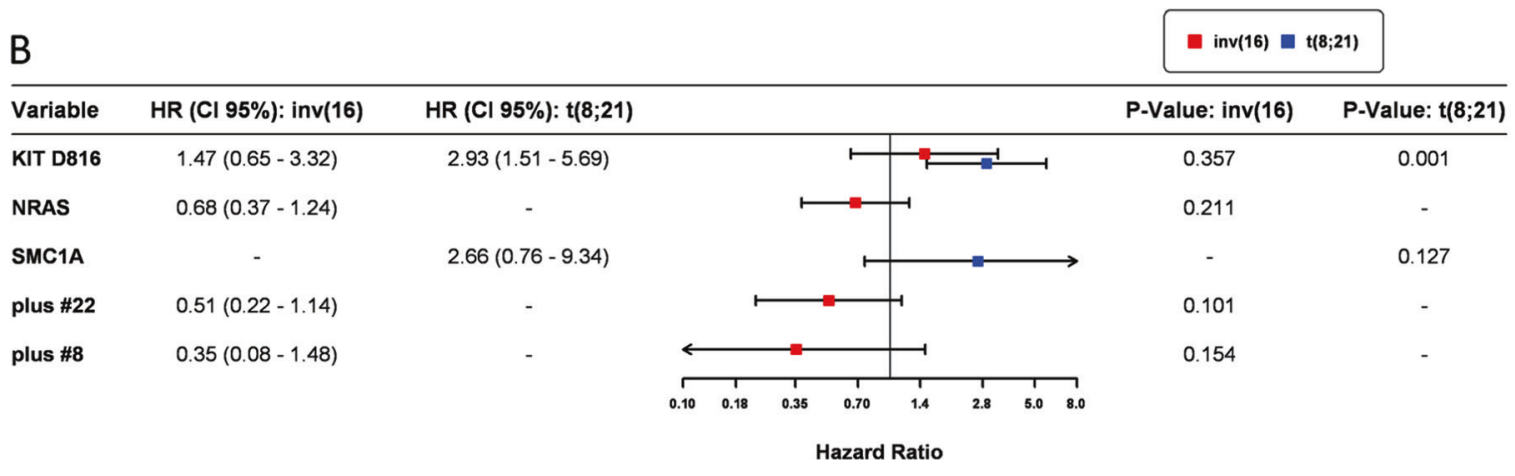

Fig. 5 Impact of selected mutations on outcome in CBF leukemia. a overall survival and $b$ relapse-free survival. Multivariate Cox regression. $\mathrm{CBFB} / \mathrm{MYH} 11$-rearranged leukemia is depicted in red, RUNX1/RUNX1T1 in blue. OS was defined from the date of treatment initiation to the date of death. Relapse-free survival was

CR achievement rates were similarly high in the inv(16) cohort $(97.1 \%)$ and the $t(8 ; 21)$ cohort $(95.3 \%)$. Furthermore, no mutation or additional chromosomal aberration was associated with $\mathrm{CR}$ in either of the two cohorts.

\section{Mutation persistence or gain at CR}

In 110 patients $\mathrm{CR}$ samples were available for detection of driver mutations, potentially indicating clonal hematopoiesis. We found persistence or gain of DNMT3A mutations in 4 out of 9 DNMT3A-mutated patients, with one patient harboring two different alterations of DNMT3A (Supplementary Table S4). Interestingly, we detected a gain of TP53 mutations during CR in three CBF leukemia patients - all of these patients eventually relapsed. In one patient, the TP53 mutation harboring clone expanded at relapse, while in the second patient the TP53 mutated clone was hardly detectable at relapse (Supplementary Table S4). For the third patient, appropriate sample material from the timepoint of relapse was not available.

\section{Discussion}

We aimed to improve our understanding of CBF leukemia, a heterogeneous AML subtype, focusing on cooperating calculated from the date of complete remission to the date of relapse or death in remission. Hazard ratios (HR), with corresponding Wald-test $p$ values and $95 \%$ confidence intervals (CI) are reported. $p$ values are considered significant at $p \leq 0.05$.

genetic events that might be responsible for the divergent clinical course. In the present study, we provide a comprehensive mutation catalog of 292 adult CBF leukemia patients and determined the prognostic relevance of mutations in distinct genes. A comparison of these intensively treated patients is conclusive even if they were treated in different trials, since all intensive AML chemotherapy protocols are similarly based on combinations of cytarabine and anthracyclines.

We found novel recurrent mutations, e.g., in NFE2 and $M N 1 . N F E 2$ is a known hematopietic transcription factor. Recently, acquired NFE2 mutations were described in 4 out of 6 patients with isolated myelosarcoma (MS) but were not found in 12 cases with MS and concomitant or previous AML [22]. Of note, one of these acquired mutations affected amino acid R272 - the mutational hotspot detected in our CBF leukemia cohort. This region (AA 271-273) within the DBD encodes the nuclear localization sequence, critical for transcriptional activation of the NFE2 protein [23]. In 2-3\% of myeloproliferative neoplasms cases, truncating NFE2 mutations were identified and confer a proliferative advantage [24]. MN1 is known as tumor suppressor gene [5]. Overexpression of $M N 1$ is an important cooperating event in inv(16) leukemia [25]. In AML with normal cytogenetics, $M N 1$ overexpression is associated with poor prognosis [26]. Of note, we identified hotspot 
mutations resulting in loss of the C-terminal part of MN1. Interestingly, it was shown that $\mathrm{C}$-terminal deletion mutants of $M N 1$ are still able to cause leukemia in mice and confer higher engraftment rates [27]. Thus, the truncating mutations found in our patients (Fig. 4) likely result in gain-offunction.

In addition, we found recurrent mutations in CSF3R and $D H X 15$. Acquisition of extracellular point mutations and mutations that truncate the cytoplasmic domain of $C S F 3 R$ is a common phenomenon in severe congenital neutropenia (SCN) and chronic neutrophilic leukemia patients and leads to ligand-independent activation of the receptor and thus, to JAK-STAT, MAPK-ERK, and PI3K-AKT signaling pathway activation $[28,29]$. SCN patients with $C S F 3 R$ mutation are predisposed to AML and the mutation frequency increases up to $80 \%$ after leukemic transformation. CSF3R mutations have been reported in $2 \%$ of pediatric AML, where they were associated with $C E B P A$ mutations and CBF-rearrangements [30]. So far, CSF3R aberrations were rarely found in adult de novo AML [29, 31]. A recent study from our research group confirmed the association of CSF3R alterations with biallelic CEBPA mutations also in adults [32]. DHX15 mutations are common in RUNX1/RUNX1T1 leukemia. DHX15 is part of the splicing machinery and missense mutations at position R222 result in increased numbers of alternative splicing events $[18,33]$. Of note, alterations in other spliceosome components are rare in CBF leukemia.

Our findings suggest that the mutatome of CBF leukemia is genetically complex with the co-existence of distinct subclones in more than half of the patients. In regards to recently published data on the prognostic impact of clonal interference in CBF leukemia [34], parallel evolution of more than one mutation in the signaling genes KIT, RAS, FLT3, JAK1/2, CBL, or PTPN11 in a single patient (assuming that these mutations arise in independent subclones) did not affect overall or RFS in our CBF leukemia cohort (Supplementary Figs. S5 and S6).

While $C B F B / M Y H 11$-rearranged leukemias were associated with mutations in $R A S$-genes, FLT3 and exon 8 frameshift mutations in KIT gene, RUNX1/RUNXIT1-rearranged leukemia correlated with mutations in KIT D816, epigenetic modifiers (ASXL1/2, TET2, IDH2, DNMT3A), cohesion complex components (RAD21, SMC3, SMC1A, STAG2), ZBTB7A, GATA2, JAK2/3 and DHX15. These recurrent mutations likely represent other pathways that are relevant for RUNX1/RUNXIT1-initiated leukemogenesis. Of note, most of these mutations have been associated with unfavorable prognosis in AML in recent studies [14, 16, 17, 35-38]. In our RUNX1/RUNX1T1 cohort, aberrations in DHX15 and $S M C 1 A$ were found to be strong and independent adverse prognostic factors for OS and RFS.

SMC1A mutations were found mostly with high allelic frequency, likely representing the predominant clone.
Although SMC1A is localized on the $\mathrm{X}$ chromosome, its alterations are neither significantly associated with $\mathrm{X}$ loss nor with gender in our cohort (Supplementary Fig. S9).

In the present study, TET2 alterations were mutually exclusive with mutations in genes of the cohesion complex and $Z B T B 7 A$. Instead they often co-occur with signaling mutations. TET2 alterations were commonly found in the predominant leukemic clone suggesting an early lesion and a potential initiating event during leukemogenesis. The tendency toward unfavorable prognosis in patients with TET2 mutation is based on the strong correlation with older age (HR = 4.25; Fig. 3). TET2 mutations were not found in patient younger than 40 years (Supplementary Fig. S10).

Compared with recently published whole-exome, wholegenome, and targeted sequencing data of heterogeneous cohorts of adult and pediatric CBF AML patients from two different research groups [8, 18], we observed a higher prevalence of alterations in signaling pathway genes, in ZBTB7A, JAK and epigenetic modifiers, a discrepancy partially explained by the often low VAF of signaling pathway mutations and the deeper sequencing coverage achieved by our targeted sequencing approach. A second potential bias might be that in both studies pooled data of adult and childhood CBF AML were analyzed. As observed by the authors themselves, adult and pediatric patients with CBF AML differ in the pattern of mutations which are dependent on age (TET2, KIT, EZH2, KDM6A, and FLT3) [35, 39]. A further limitation was mentioned by Faber et al. themselves: despite adequate sequence coverage in their WES cohort they did not identify mutations in ASXL1 [18]. Moreover, the study by Duployez et al. was limited to mutational hotspots in 40 genes, recurrently mutated in myeloid malignancies [8]. Thus, they missed novel mutations in genes like ZBTB7A, MN1, and NFE2, as well as novel mutational hotspots like N676K in exon 16 of FLT3.

A limitation of our study is that we focused on a defined set of known and novel genes. The entire coding sequence (exome) was analyzed only for $12 \mathrm{CBF}$ patients. This might be the reason why we missed novel recurring but rare mutations in RUNX1/RUNXIT1-rearranged leukemia [CCND2 (6-12\%) [18, 40], MGA (8\%) [18]]. However, whole-exome or whole-genome sequencing requires matched germline samples, and suitable DNA specimens were not available for all of our patients.

In summary, our study provides a detailed overview of cooperating mutations in one of the largest adult CBF leukemia cohorts so far and highlights fundamental differences between $C B F B / M Y H 11$ - and RUNX1/RUNX1T1-rearranged leukemia. Thus, they seem to represent distinct clinicobiological entities. We provide detailed insights into the prognostic relevance of several cooperating mutations and found a significant impact on survival for mutations in KIT, DHX15, and SMC1A and loss of the Y-chromosome. Our 
findings have implications for the use of already established targeted therapies aimed at eradicating malignant clones carrying mutations in FLT3, KIT, JAK2, TET2, DNMT3A, and $I D H I / 2$. Furthermore, the unfavorable genetic profiles in CBF leukemia patients could be considered for future risk stratification. Especially for the patients with high risk of relapse, the clinical mutatome of CBF leukemia has a strong potential for translation into alternative treatment strategies.

Acknowledgements We thank the patients and participating centers of the German Acute Myeloid Leukemia Cooperative Group (AMLCG) of the Study Alliance Leukemia (SAL). This work was supported by grants from the Wilhelm Sander-Stiftung (2013.086.2 to TH and 2014.162.2 to PG). KHM, KS, PAG., WH and HB acknowledge support from the German Research Foundation (DFG) within the Collaborative Research Centre (SFB) 1243 'Cancer Evolution' (projects A06, A07, $\mathrm{A} 08$, and Z02). The statistical analysis was funded partly by the Jose Carreras Foundation (DJCLS H 09/01f, to DG). SKB is supported by the Leukaemia \& Blood Cancer New Zealand and the family of Marijanna Kumerich. TH received a Physician Scientist Grant (G-509200004) from the Helmholtz Zentrum München.

Author contributions $\mathrm{SO}, \mathrm{SKB}$, and PAG conceived and designed the experiments; SO, BK, KB, and SK performed experiments; SO, KHM, $\mathrm{SAB}$, and $\mathrm{TH}$ analyzed the data; KHM, AG and SV provided the bioinformatics support; HB managed the sequencing platform; SO, BK, NPK, CT, JMM, FS, SS, SKB, and KS characterized patient samples; MCS, DG, JB, WEB, BJW, and WH coordinated the German Acute Myeloid Leukemia Cooperative Group clinical trials; CR, JS, $\mathrm{AK}$, and GE coordinated the SAL clinical trials; PAG, KS and SKB supervised the project; and SO, SKB, and PAG wrote the paper.

\section{Compliance with ethical standards}

Conflict of interest The authors declare that they have no conflict of interest.

Publisher's note Springer Nature remains neutral with regard to jurisdictional claims in published maps and institutional affiliations.

Open Access This article is licensed under a Creative Commons Attribution 4.0 International License, which permits use, sharing, adaptation, distribution and reproduction in any medium or format, as long as you give appropriate credit to the original author(s) and the source, provide a link to the Creative Commons license, and indicate if changes were made. The images or other third party material in this article are included in the article's Creative Commons license, unless indicated otherwise in a credit line to the material. If material is not included in the article's Creative Commons license and your intended use is not permitted by statutory regulation or exceeds the permitted use, you will need to obtain permission directly from the copyright holder. To view a copy of this license, visit http://creativecommons. org/licenses/by/4.0/.

\section{References}

1. Speck NA, Gilliland DG. Core-binding factors in haematopoiesis and leukaemia. Nat Rev Cancer. 2002;2:502-13.

2. Döhner H, Estey E, Grimwade D, Amadori S, Appelbaum FR, Büchner T, et al. Diagnosis and management of AML in adults:
2017 ELN recommendations from an international expert panel. Blood. 2017;129:424.

3. Opatz S, Polzer H, Herold T, Konstandin NP, Ksienzyk B, Zellmeier E, et al. Exome sequencing identifies recurring FLT3 N676K mutations in core-binding factor leukemia. Blood. 2013; 122:1761-9.

4. Haferlach C, Dicker F, Kohlmann A, Schindela S, Weiss T, Kern W, et al. AML with CBFB-MYH11 rearrangement demonstrate RAS pathway alterations in $92 \%$ of all cases including a high frequency of NF1 deletions. Leukemia. 2010;24:1065-9.

5. Paschka P, Döhner K. Core-binding factor acute myeloid leukemia: can we improve on HiDAC consolidation? ASH Educ Program Book. 2013;2013:209-19.

6. Paschka P, Du J, Schlenk RF, Gaidzik VI, Bullinger L, Corbacioglu A, et al. Secondary genetic lesions in acute myeloid leukemia with inv(16) or $\mathrm{t}(16 ; 16)$ : a study of the German-Austrian AML Study Group (AMLSG). Blood. 2013;121:170-7.

7. Paschka P, Marcucci G, Ruppert AS, Mrozek K, Chen H, Kittles RA, et al. Adverse prognostic significance of KIT mutations in adult acute myeloid leukemia with inv(16) and $\mathrm{t}(8 ; 21)$ : a Cancer and Leukemia Group B Study. J Clin Oncol. 2006;24:3904-11.

8. Duployez N, Marceau-Renaut A, Boissel N, Petit A, Bucci M, Geffroy S, et al. Comprehensive mutational profiling of core binding factor acute myeloid leukemia. Blood. 2016;127:2451.

9. Marcucci G, Mrozek K, Ruppert AS, Maharry K, Kolitz JE, Moore JO, et al. Prognostic factors and outcome of core binding factor acute myeloid leukemia patients with $\mathrm{t}(8 ; 21)$ differ from those of patients with inv(16): a Cancer and Leukemia Group B study. J Clin Oncol. 2005;23:5705-17.

10. Schlenk RF, Benner A, Krauter J, Buchner T, Sauerland C, Ehninger $\mathrm{G}$, et al. Individual patient data-based meta-analysis of patients aged 16 to 60 years with core binding factor acute myeloid leukemia: a survey of the German Acute Myeloid Leukemia Intergroup. J Clin Oncol. 2004;22:3741-50.

11. Appelbaum FR, Kopecky KJ, Tallman MS, Slovak ML, Gundacker HM, Kim HT, et al. The clinical spectrum of adult acute myeloid leukaemia associated with core binding factor translocations. Br J Haematol. 2006;135:165-73.

12. Hartmann L, Dutta S, Opatz S, Vosberg S, Reiter K, Leubolt G, et al. ZBTB7A mutations in acute myeloid leukaemia with $\mathrm{t}(8 ; 21)$ translocation. Nat Commun. 2016;7:11733.

13. Greif PA, Dufour A, Konstandin NP, Ksienzyk B, Zellmeier E, Tizazu B, et al. GATA2 zinc finger 1 mutations associated with biallelic CEBPA mutations define a unique genetic entity of acute myeloid leukemia. Blood. 2012;120:395-403.

14. Metzeler KH, Herold T, Rothenberg-Thurley M, Amler S, Sauerland MC, Görlich D, et al. Spectrum and prognostic relevance of driver gene mutations in acute myeloid leukemia. Blood. 2016;128:686.

15. Mrozek K, Marcucci G, Paschka P, Bloomfield CD. Advances in molecular genetics and treatment of core-binding factor acute myeloid leukemia. Curr Opin Oncol. 2008;20:711-8.

16. Micol J-B, Duployez N, Boissel N, Petit A, Geffroy S, Nibourel $\mathrm{O}$, et al. Frequent ASXL2 mutations in acute myeloid leukemia patients with $\mathrm{t}(8 ; 21) / \mathrm{RUNX} 1-\mathrm{RUNX} 1 \mathrm{~T} 1$ chromosomal translocations. Blood. 2014;124:1445-9.

17. Krauth MT, Eder C, Alpermann T, Bacher U, Nadarajah N, Kern $\mathrm{W}$, et al. High number of additional genetic lesions in acute myeloid leukemia with $t(8 ; 21) / R U N X 1-R U N X 1 T 1$ : frequency and impact on clinical outcome. Leukemia. 2014;28:1449-58.

18. Faber ZJ, Chen X, Gedman AL, Boggs K, Cheng J, Ma J, et al. The genomic landscape of core-binding factor acute myeloid leukemias. Nat Genet. 2016;48:1551-6.

19. Lavallée V-P, Lemieux S, Boucher G, Gendron P, Boivin I, Armstrong RN, et al. RNA-sequencing analysis of core binding 
factor AML identifies recurrent ZBTB7A mutations and defines RUNX1-CBFA2T3 fusion signature. Blood. 2016;127:2498.

20. Christen F, Hoyer K, Yoshida K, Hou H-A, Waldhueter N, Heuser $\mathrm{M}$, et al. Genomic landscape and clonal evolution of acute myeloid leukemia with $\mathrm{t}(8 ; 21)$ : an international study on 331 patients. Blood. 2019;133:1140-51.

21. Allen C, Hills RK, Lamb K, Evans C, Tinsley S, Sellar R, et al. The importance of relative mutant level for evaluating impact on outcome of KIT, FLT3 and CBL mutations in core-binding factor acute myeloid leukemia. Leukemia. 2013;27:1891-901.

22. Lazarevic V, Orsmark-Pietras C, Lilljebjörn H, Pettersson L, Rissler $\mathrm{M}$, Lübking $\mathrm{A}$, et al. Isolated myelosarcoma is characterized by recurrent NFE2 mutations and concurrent preleukemic clones in the bone marrow. Blood. 2018;131:577.

23. Perdomo J, Fock EL, Kaur G, Yan F, Khachigian LM, Jans DA, et al. A monopartite sequence is essential for p45 NF-E2 nuclear translocation, transcriptional activity and platelet production. J Thrombosis Haemost. 2010;8:2542-53.

24. Jutzi JS, Bogeska R, Nikoloski G, Schmid CA, Seeger TS, Stegelmann F, et al. MPN patients harbor recurrent truncating mutations in transcription factor NF-E2. J Exp Med. 2013;210: 1003-19.

25. Carella C, Bonten J, Sirma S, Kranenburg TA, Terranova S, Klein-Geltink R, et al. MN1 overexpression is an important step in the development of inv(16) AML. Leukemia. 2007;21:1679-90.

26. Heuser M, Beutel G, Krauter J, Dohner K, von Neuhoff N, Schlegelberger B, et al. High meningioma 1 (MN1) expression as a predictor for poor outcome in acute myeloid leukemia with normal cytogenetics. Blood. 2006;108:3898-905.

27. Lai CK, Moon Y, Kuchenbauer F, Starzcynowski DT, Argiropoulos B, Yung E, et al. Cell fate decisions in malignant hematopoiesis: leukemia phenotype is determined by distinct functional domains of the MN1 oncogene. PLOS ONE. 2014;9: e112671.

28. Ward AC, van Aesch YM, Schelen AM, Touw IP. Defective internalization and sustained activation of truncated granulocyte colony-stimulating factor receptor found in severe congenital neutropenia/acute myeloid leukemia. Blood. 1999;93:447.

29. Beekman R, Valkhof M, van Strien P, Valk PJM, Touw IP. Prevalence of a new auto-activating colony stimulating factor 3 receptor mutation (CSF3R-T595I) in acute myeloid leukemia and severe congenital neutropenia. Haematologica. 2013;98:e62.
30. Maxson JE, Ries RE, Wang Y-C, Gerbing RB, Kolb EA, Thompson SL, et al. CSF3R mutations have a high degree of overlap with CEBPA mutations in pediatric AML. Blood. 2016; 127:3094.

31. Zhang H, Means S, Schultz AR, Watanabe-Smith K, Medeiros BC, Bottomly D, et al. Unpaired extracellular cysteine mutations of CSF3R mediate gain or loss of function. Cancer Res. 2017;77:4258.

32. Konstandin NP, Pastore F, Herold T, Dufour A, RothenbergThurley M, Hinrichsen T, et al. Genetic heterogeneity of cytogenetically normal AML with mutations of CEBPA. Blood Adv. 2018;2:2724.

33. Sood R, Hansen NF, Donovan FX, Carrington B, Bucci D, Maskeri B, et al. Somatic mutational landscape of AML with inv (16) or $\mathrm{t}(8 ; 21)$ identifies patterns of clonal evolution in relapse leukemia. Leukemia. 2016;30:501-4.

34. Itzykson R, Duployez N, Fasan A, Decool G, Marceau-Renaut A, Meggendorfer $\mathrm{M}$, et al. Clonal interference of signaling mutations worsens prognosis in core-binding factor acute myeloid leukemia. Blood. 2018;132:187.

35. Metzeler KH, Maharry K, Radmacher MD, Mrózek K, Margeson D, Becker H, et al. TET2 mutations improve the new European LeukemiaNet risk classification of acute myeloid leukemia: a Cancer and Leukemia Group B study. J Clin Oncol. 2011;29:1373-81.

36. Cher CY, Leung GM, Au CH, Chan TL, Ma ES, Sim JP, et al. Next-generation sequencing with a myeloid gene panel in corebinding factor AML showed KIT activation loop and TET2 mutations predictive of outcome. Blood cancer J. 2016;6:e442.

37. Illmer T, Schaich M, Ehninger G, Thiede C. Tyrosine kinase mutations of JAK2 are rare events in AML but influence prognosis of patients with CBF-leukemias. Haematologica. 2007;92:137-8.

38. Dohner K, Du J, Corbacioglu A, Scholl C, Schlenk RF, Dohner H. JAK2V617F mutations as cooperative genetic lesions in $\mathrm{t}(8 ; 21)$ positive acute myeloid leukemia. Haematologica. 2006;91:1569-70.

39. Busque L, Patel JP, Figueroa ME, Vasanthakumar A, Provost S, Hamilou Z, et al. Recurrent somatic TET2 mutations in normal elderly individuals with clonal hematopoiesis. Nat Genet. 2012; 44:1179-81.

40. Eisfeld AK, Kohlschmidt J, Schwind S, Nicolet D, Blachly JS, Orwick S, et al. Mutations in the CCND1 and CCND2 genes are frequent events in adult patients with $\mathrm{t}(8 ; 21)(\mathrm{q} 22 ; \mathrm{q} 22)$ acute myeloid leukemia. Leukemia. 2017;31:1278-85. 